Теорія Ймовір. та Матем. Статист. Вип. 76, 2007
Theor. Probability and Math. Statist.

No. 76, 2008, Pages 41-48

S 0094-9000(08)00730-8

Article electronically published on July 10, 2008

\title{
ON THE ASYMPTOTIC DEGENERATION OF SYSTEMS OF LINEAR INHOMOGENEOUS STOCHASTIC DIFFERENTIAL EQUATIONS
}

UDC 519.21

\author{
OLEKSANDER IL'CHENKO
}

\begin{abstract}
Assuming the almost sure stability of a linear homogeneous system, we obtain sufficient conditions for the convergence to zero, in probability as well as pathwise, of solutions of the system of linear inhomogeneous stochastic differential equations.
\end{abstract}

It is well known in the theory of deterministic systems that solutions of a linear inhomogeneous system approach zero if the homogeneous part of the system is exponentially stable and the inhomogeneous terms converge to zero as time goes to infinity. A similar result is considered in the current paper for the stochastic case. The author gives sufficient conditions for the convergence to zero, in probability and with probability one, of solutions of an inhomogeneous system of differential equations if the stochastic semigroup generated by the linear homogeneous part is stable with probability one.

Consider a system of stochastic differential equations

$$
d x(t)=\left(A_{0} x(t)+f_{0}(t)\right) d t+\sum_{k=1}^{m}\left(A_{k} x(t)+f_{k}(t)\right) d w_{k}(t),
$$

where $A_{k}$ are $(n \times n)$ matrices;

$$
f_{k}(t)=\left(f_{k 1}(t), \ldots, f_{k n}(t)\right), \quad t \geq 0,
$$

are vector functions; $w_{r}(t), t \geq 0$, are independent one-dimensional Wiener processes;

$$
x(t)=\left(x_{1}(t), \ldots, x_{n}(t)\right), \quad t \geq 0,
$$

is a solution written as a row vector.

Denote by $\left\{e_{i}\right\}_{i=1}^{n}$ an orthonormal basis in $\mathbb{R}^{n}$ and by

$$
\langle x, y\rangle=\sum_{i=1}^{n} x_{i} y_{i} \quad \text { and } \quad\|x\|=\langle x, x\rangle^{1 / 2}
$$

the scalar product and norm of vectors in $\mathbb{R}^{n}$, respectively.

Let $H_{s}^{t}$ be a stochastic semigroup of nondegenerate operators,

$$
H_{s}^{t}=H_{r}^{t} H_{s}^{r}, \quad 0 \leq s \leq r \leq t,
$$

such that (see [7])

$$
d H_{s}^{t}=A_{0} H_{s}^{t} d t+\sum_{k=1}^{m} A_{k} H_{s}^{t} d w_{k}(t), \quad H_{s}^{s}=I, s \leq t .
$$

2000 Mathematics Subject Classification. Primary 60H10; Secondary 34F05. 
OLEKSANDER IL'CHENKO

We assume that the semigroup $H_{s}^{t}$ is stable with probability one; that is,

$$
\mathrm{P}\left\{\lim _{t \rightarrow+\infty} H_{0}^{t} x=0\right\}=1 \quad \text { for all } x \in \mathbb{R}^{n} .
$$

It is known (see [5, 7]) that the stability with probability one of the semigroup $H_{s}^{t}$ is equivalent to the exponential $p$-stability of $H_{s}^{t}$ for sufficiently small $p>0$; that is, there are constants $D=D(p)>0$ and $\lambda=\lambda(p)>0$ such that

$$
\sup _{\|x\|=1} \mathrm{E}\left\|H_{s}^{t} x\right\|^{p} \leq D e^{-\lambda p(t-s)}, \quad p \in\left(0, p_{0}\right) .
$$

A solution $x(t), t \geq 0$, of system (1) can be represented in the following form:

$$
x(t)=H_{0}^{t} x+\int_{0}^{t}\left(H_{u}^{t}\right)\left(f_{0}(u)-\sum_{k=1}^{m} A_{k} f_{k}(u)\right) d u+\sum_{k=1}^{m} H_{0}^{t} \int_{0}^{t}\left(H_{0}^{u}\right)^{-1} f_{k}(u) d w_{k}(u)
$$

(see [4, 6]). To study the behavior of $x(t)$ as $t \rightarrow+\infty$, one needs to estimate the distributions of terms on the right hand side of (3). The following two auxiliary results contain necessary estimates.

Lemma 1. Let condition (2) hold. If $\varphi(t), t \geq 0$, is a continuous vector function, then, for arbitrary $\varepsilon>0$ and $\theta>0$, there exists a constant $\mathcal{L}<+\infty$ such that

$$
\mathrm{P}\left\{\sup _{k \leq t \leq k+1}\left\|\int_{0}^{t} H_{u}^{t} \varphi(u) d u\right\|>\varepsilon\right\} \leq \mathcal{L} \sum_{i=0}^{k} e^{-(\lambda-\theta)(k-i) p}\left(\sup _{i \leq u \leq i+1}\|\varphi(u)\|\right)^{p}
$$

for all $k \in \mathbb{Z}_{+}$.

Proof of Lemma 1. We make use of the following obvious inequalities:

$$
\begin{gathered}
M \sum_{i=0}^{k} e^{-\theta(k+1-i)}<1, \quad M=e^{\theta}\left(1-e^{-\theta}\right), \theta>0, k \in \mathbb{Z}_{+}, \\
\left\|H_{s}^{t} \zeta\right\| \leq \sum_{i=1}^{n}\left\|H_{s}^{t} e_{i}\right\|\|\zeta\|, \quad \zeta=\zeta(\omega) \in \mathbb{R}^{n} .
\end{gathered}
$$

Taking into account (4) we obtain the bound

$$
\begin{aligned}
& \mathrm{P}\left\{\sup _{k \leq t \leq k+1}\left\|\int_{0}^{t} H_{u}^{t} \varphi(u) d u\right\|>\varepsilon\right\} \\
& \leq \mathrm{P}\left\{\sup _{k \leq t \leq k+1}\left\|\sum_{i=0}^{k-1} H_{i+1}^{t} \int_{i}^{i+1} H_{u}^{i+1} \varphi(u) d u+\int_{k}^{t} H_{u}^{t} \varphi(u) d u\right\|>\varepsilon\right\} \\
& \leq \sum_{i=0}^{k-1} \mathrm{P}\left\{\sup _{k \leq t \leq k+1}\left\|H_{i+1}^{t} \int_{i}^{i+1} H_{u}^{i+1} \varphi(u) d u\right\|>M e^{-\theta(k+1-i)} \varepsilon\right\} \\
&+\mathrm{P}\left\{\sup _{k \leq t \leq k+1}\left\|\int_{k}^{t} H_{u}^{t} \varphi(u) d u\right\|>M e^{-\theta} \varepsilon\right\} .
\end{aligned}
$$

Put $M_{i}^{k}=M e^{-\theta(k+1-i)} \varepsilon$ and $\varphi_{i}=\sup _{i \leq u \leq i+1}\|\varphi(u)\|, i=0, \ldots, k$. Lemma 1 follows from the following inequalities:

$$
\begin{gathered}
\mathrm{P}\left\{\sup _{k \leq t \leq k+1}\left\|H_{i+1}^{t} \int_{i}^{i+1} H_{u}^{i+1} \varphi(u) d u\right\|>M_{i}^{k}\right\} \leq \mathcal{L}_{1} e^{-(\lambda-\theta)(k-i) p} \varphi_{i}^{p}, \\
\mathrm{P}\left\{\sup _{k \leq t \leq k+1}\left\|\int_{k}^{t} H_{u}^{t} \varphi(u) d u\right\|>M_{k}^{k}\right\} \leq \mathcal{L}_{2} \varphi_{k}^{p} .
\end{gathered}
$$


Indeed, if both bounds (7) and (8) hold, then we use (6) and choose $\mathcal{L}=\max \left\{\mathcal{L}_{1}, \mathcal{L}_{2}\right\}$, whence Lemma 1 follows.

Now we prove (77). Let $N_{i}^{k}=n^{-3} M_{i}^{k}$. Using (5) we get

$$
\begin{aligned}
\mathrm{P}\left\{\sup _{k \leq t \leq k+1}\left\|H_{i+1}^{t} \int_{i}^{i+1} H_{u}^{i+1} \varphi(u) d u\right\|>M_{i}^{k}\right\} \\
\quad \leq \sum_{l, m, j=1}^{n} \mathrm{P}\left\{\sup _{k \leq t \leq k+1}\left\|H_{k}^{t} e_{l}\right\| \cdot\left\|H_{i+1}^{k} e_{m}\right\| \sup _{i \leq u \leq i+1}\left\|H_{u}^{i+1} e_{j}\right\| \varphi_{i}>n^{-3} M_{i}^{k}\right\} \\
\quad \leq \sum_{l, m, j=1}^{n}\left(N_{i}^{k}\right)^{-p} \mathrm{E}\left(\sup _{k \leq t \leq k+1}\left\|H_{k}^{t} e_{l}\right\|\right)^{p} \mathrm{E}\left\|H_{i+1}^{k} e_{m}\right\|^{p} \mathrm{E}\left(\sup _{i \leq u \leq i+1}\left\|H_{u}^{i+1} e_{j}\right\|\right)^{p} \varphi_{i}^{p} \\
\leq n^{3}\left(N_{i}^{k}\right)^{-p} L_{0} D e^{-\lambda p(k-i-1)} L_{1} \varphi_{i}^{p} \leq \mathcal{L}_{1} e^{-(\lambda-\theta)(k-i) p} \varphi_{i}^{p},
\end{aligned}
$$

where

$$
\begin{gathered}
\mathcal{L}_{1}=n^{3} L_{0} D L_{1}\left(n^{-3} M \varepsilon e^{-(\lambda+\theta)}\right)^{-p}, \quad L_{0}=\sup _{\|x\|=1} \mathrm{E}\left(\sup _{0 \leq t \leq 1}\left\|H_{0}^{t} x\right\|\right)^{p}, \\
L_{1}=\sup _{\|x\|=1} \mathrm{E}\left(\sup _{0 \leq t \leq 1}\left\|H_{t}^{1} x\right\|\right)^{p} .
\end{gathered}
$$

The proof of (8) is analogous. Indeed,

$$
\begin{aligned}
\mathrm{P}\left\{\sup _{k \leq t \leq k+1}\left\|\int_{k}^{t} H_{u}^{t} \varphi(u) d u\right\|>M_{k}^{k}\right\} \\
\quad \leq \mathrm{P}\left\{\sup _{0 \leq u \leq v \leq 1}\left\|\left(H_{v}^{1}\right)^{-1} H_{u}^{1} \varphi(k+u)\right\|>M_{k}^{k}\right\} \\
\quad \leq \sum_{l, m=1}^{n}\left(n N_{k}^{k}\right)^{-p} \mathrm{E}\left\{\left(\sup _{0 \leq v \leq 1}\left\|\left(H_{v}^{1}\right)^{-1} e_{l}\right\|\right)^{p}\left(\sup _{0 \leq u \leq 1}\left\|H_{u}^{1} e_{m}\right\|\right)^{p}\right\} \varphi_{k}^{p} \\
\quad \leq \sum_{l, m=1}^{n}\left(n N_{k}^{k}\right)^{-p}\left\{\mathrm{E}\left(\sup _{0 \leq v \leq 1}\left\|\left(H_{v}^{1}\right)^{-1} e_{l}\right\|\right)^{2 p}\right\}^{1 / 2}\left\{\mathrm{E}\left(\sup _{0 \leq u \leq 1}\left\|H_{u}^{1} e_{m}\right\|\right)^{2 p}\right\}^{1 / 2} \varphi_{k}^{p} \\
\leq \mathcal{L}_{2} \varphi_{k}^{p},
\end{aligned}
$$

where

$$
\begin{gathered}
\mathcal{L}_{2}=\left(n^{-2} M_{k}^{k}\right)^{-p} n^{2}\left(L_{3}\right)^{1 / 2}\left(L_{4}\right)^{1 / 2}, \quad L_{3}=\sup _{\|x\|=1} \mathrm{E}\left(\sup _{0 \leq t \leq 1}\left\|\left(H_{t}^{1}\right)^{-1} x\right\|\right)^{2 p}, \\
L_{4}=\sup _{\|x\|=1} \mathrm{E}\left(\sup _{0 \leq t \leq 1}\left\|H_{t}^{1} x\right\|\right)^{2 p} .
\end{gathered}
$$

Lemma 1 is proved.

Lemma 2. Let condition (2) hold. If $\varphi(t), t \geq 0$, is a continuous vector function, then, for all $\varepsilon>0$ and $\theta>0$, there exists a constant $\mathcal{K}<+\infty$ such that

$$
\begin{gathered}
\mathrm{P}\left\{\sup _{k \leq t \leq k+1}\left\|H_{0}^{t} \int_{0}^{t}\left(H_{0}^{u}\right)^{-1} \varphi(u) d w_{r}(u)\right\|>\varepsilon\right\} \\
\leq \mathcal{K} \sum_{i=0}^{k} e^{-(\lambda-\theta)(k-i) p}\left(\sup _{i \leq u \leq i+1}\|\varphi(u)\|\right)^{p}
\end{gathered}
$$

for all $k \in \mathbb{Z}_{+}$and $r=1, \ldots, m$. 
Proof of Lemma 2. It is clear that

$$
\begin{aligned}
\mathrm{P}\left\{\sup _{k \leq t \leq k+1}\left\|H_{0}^{t} \int_{0}^{t}\left(H_{0}^{u}\right)^{-1} \varphi(u) d w_{r}(u)\right\|>2 \varepsilon\right\} \\
\leq \mathrm{P}\left\{\sup _{k \leq t \leq k+1}\left\|H_{0}^{t} \int_{0}^{k}\left(H_{0}^{u}\right)^{-1} \varphi(u) d w_{r}(u)\right\|>\varepsilon\right\} \\
+\mathrm{P}\left\{\sup _{k \leq t \leq k+1}\left\|H_{0}^{t} \int_{k}^{t}\left(H_{0}^{u}\right)^{-1} \varphi(u) d w_{r}(u)\right\|>\varepsilon\right\} .
\end{aligned}
$$

Probabilities (9) and (10) are estimated separately. We start with (9). Using the equality

$$
H_{s}^{v} \int_{s}^{t}\left(H_{s}^{u}\right)^{-1} \varphi(u) d w_{r}(u)=-\int_{t}^{s} H_{u}^{v} \varphi(u) d w_{r}(u)-\int_{t}^{s} H_{u}^{v} A_{r} \varphi(u) d u
$$

proved in [3, 4, for $s \leq t \leq v$, we obtain

$$
\begin{aligned}
\mathrm{P}\left\{\sup _{k \leq t \leq k+1}\left\|H_{0}^{t} \int_{0}^{k}\left(H_{0}^{u}\right)^{-1} \varphi(u) d w_{r}(u)\right\|>2 \varepsilon\right\} \\
\leq \mathrm{P}\left\{\sup _{k \leq t \leq k+1}\left\|\int_{k}^{0} H_{u}^{t} \varphi(u) d w_{r}(u)\right\|>\varepsilon\right\} \\
+\mathrm{P}\left\{\sup _{k \leq t \leq k+1}\left\|\int_{k}^{0} H_{u}^{t} A_{r} \varphi(u) d u\right\|>\varepsilon\right\} .
\end{aligned}
$$

Probability (12) is estimated with the help of Lemma 1. It remains to prove a corresponding inequality for probability (11).

Probability (11) is estimated similarly to Lemma 1 . Following the ideas of 3 , 4 , we use an inequality for the moments of stochastic integrals (see [2]). Put $\phi_{i}(u)=H_{u}^{i+1} \varphi(u)$,

$$
L_{0}=\sup _{\|x\|=1} \mathrm{E}\left(\sup _{0 \leq t \leq 1}\left\|H_{0}^{t} x\right\|\right)^{p}
$$

and $M_{i}^{k}=M e^{-\theta(k+1-i)} \varepsilon$. We have

$$
\begin{aligned}
\mathrm{P}\left\{\sup _{k \leq t \leq k+1}\left\|\int_{k}^{0} H_{u}^{t} \varphi(u) d w_{r}(u)\right\|>\varepsilon\right\} \\
\quad \leq \sum_{i=0}^{k-1} \sum_{l, m, j=1}^{n} \mathrm{P}\left\{\sup _{k \leq t \leq k+1}\left\|H_{k}^{t} e_{l}\right\| \cdot\left\|H_{i+1}^{k} e_{m}\right\|\left|\int_{i+1}^{i}\left(\phi_{i}(u)\right)_{j} d w_{r}(u)\right|>n^{-3} M_{i}^{k}\right\} \\
\leq \sum_{i=0}^{k-1} \sum_{j=1}^{n} n^{2}\left(n^{-3} M_{i}^{k}\right)^{-p} L_{0} D e^{-\lambda(k-i-1) p} \mathrm{E}\left\{\sup _{i \leq v \leq i+1}\left|\int_{i+1}^{v}\left(\phi_{i}(u)\right)_{j} d w_{r}(u)\right|\right\}^{p} \\
\leq \sum_{i=0}^{k-1} \sum_{j=1}^{n} n^{2}\left(n^{-3} M_{i}^{k}\right)^{-p} L_{0} D e^{-\lambda(k-i-1) p} c_{p} \mathrm{E}\left\{\int_{i}^{i+1}\left(\phi_{i}(u)\right)_{j}^{2} d u\right\}^{p / 2} \\
\leq n^{3} L_{0} D\left(n^{-3} M \varepsilon e^{-(\lambda+\theta)}\right)^{-p} c_{p} \sum_{i=0}^{k-1} e^{-(\lambda-\theta)(k-i) p} \mathrm{E}\left\{\int_{i}^{i+1}\left\|H_{u}^{i+1} \varphi(u)\right\|^{2} d u\right\}^{p / 2} .
\end{aligned}
$$

The estimate desired for probability (11) follows from the inequality

$$
\mathrm{E}\left\{\int_{i}^{i+1}\left\|H_{u}^{i+1} \varphi(u)\right\|^{2} d u\right\}^{p / 2} \leq \mathcal{K}_{1}\left(\sup _{i \leq u \leq i+1}\|\varphi(u)\|\right)^{p} .
$$


To prove the latter inequality we put $\varphi_{i}=\sup _{i \leq u \leq i+1}\|\varphi(u)\|$ and $\psi(u)=\varphi(u) \varphi_{i}^{-1}$. Then

$$
\begin{aligned}
& \mathrm{E}\left\{\int_{i}^{i+1}\left\|H_{u}^{i+1} \varphi(u)\right\|^{2} d u\right\}^{p / 2} \leq \mathrm{E}\left\{\sup _{i \leq u \leq i+1}\left\|H_{u}^{i+1} \psi(u)\right\|^{2}\right\}^{p / 2} \varphi_{i}^{p} \\
& \quad \leq\left(1+\sum_{j=0}^{\infty} 2^{p(j+1)} \sum_{l=1}^{n} \mathrm{P}\left\{n^{-1} 2^{j}<\sup _{i \leq u \leq i+1}\left\|H_{u}^{i+1} e_{l}\right\|\|\psi(u)\|\right\}\right) \varphi_{i}^{p} \\
& \quad \leq\left(1+\sum_{j=0}^{\infty} 2^{p(j+1)} n\left(n^{-1} 2^{j}\right)^{-(p+\delta)} \sup _{\|x\|=1} \mathrm{E}\left\{\sup _{i \leq u \leq i+1}\left\|H_{u}^{i+1} x\right\|\right\}^{(p+\delta)}\right) \varphi_{i}^{p} \\
& \leq \mathcal{K}_{1} \varphi_{i}^{p},
\end{aligned}
$$

where

$$
\mathcal{K}_{1}=1+2^{p} n^{1+p+\delta} L_{5} \sum_{j=0}^{\infty} 2^{-\delta j}, \quad L_{5}=\sup _{\|x\|=1} \mathrm{E}\left(\sup _{0 \leq t \leq 1}\left\|H_{t}^{1} x\right\|\right)^{p+\delta}, \quad \delta>0 .
$$

Therefore we have proved for (111) that

$$
\mathrm{P}\left\{\sup _{k \leq t \leq k+1}\left\|\int_{k}^{0} H_{u}^{t} \varphi(u) d w_{r}(u)\right\|>\varepsilon\right\} \leq \mathcal{K}_{2} \sum_{i=0}^{k-1} e^{-(\lambda-\theta)(k-i) p}\left(\sup _{i \leq u \leq i+1}\|\varphi(u)\|\right)^{p} .
$$

It remains to estimate probability (10). Let $\widetilde{N}=\left(n^{-2} \varepsilon\right)^{-1}$. Then

$$
\begin{aligned}
\mathrm{P}\left\{\sup _{k \leq t \leq k+1}\left\|H_{k}^{t} \int_{k}^{t}\left(H_{k}^{u}\right)^{-1} \varphi(u) d w_{r}(u)\right\|>\varepsilon\right\} \\
\left.\quad \leq \sum_{i, j=1}^{n} \mathrm{P}\left\{\sup _{k \leq t \leq k+1}\left\|H_{k}^{t} e_{j}\right\|_{k \leq t \leq k+1}\left|\sup _{k}\right|\left(H_{k}^{u}\right)^{-1} \varphi(u)\right)_{i} d w_{r}(u) \mid>n^{-2} \varepsilon\right\} \\
\quad \leq \sum_{i, j=1}^{n} \widetilde{N}^{p} \mathrm{E}\left\{\left(\sup _{k \leq t \leq k+1}\left\|H_{k}^{t} e_{j}\right\|\right)^{p}\left(\sup _{k \leq t \leq k+1}\left|\int_{k}^{t}\left(\left(H_{k}^{u}\right)^{-1} \varphi(u)\right)_{i} d w_{r}(u)\right|\right)^{p}\right\} \\
\leq n^{2} \widetilde{N}^{p} L_{6}\left\{\mathrm{E}\left\{\int_{k}^{k+1}\left\|\left(H_{k}^{u}\right)^{-1} \varphi(u)\right\|^{2} d u\right\}^{p}\right\}^{1 / 2} \\
\quad \leq \mathcal{K}_{3}\left(\sup _{k \leq u \leq k+1}\|\varphi(u)\|\right)^{p},
\end{aligned}
$$

where

$$
L_{6}=\sup _{\|x\|=1}\left\{\mathrm{E}\left(\sup _{0 \leq t \leq 1}\left\|H_{0}^{t} x\right\|\right)^{2 p}\right\}^{1 / 2}
$$

Lemma 2 is proved.

Now we turn to the main results. Put

$$
g_{0}(t)=f_{0}(t)-\sum_{k=1}^{m} A_{k} f_{k}(t), \quad g_{i}(t)=f_{i}(t), \quad i=1, \ldots, m .
$$

Theorem 1. Let condition (2) hold and let $g_{i}(t), t \geq 0, i=0, \ldots, m$, be continuous functions such that

$$
\lim _{t \rightarrow+\infty} g_{i}(t)=0
$$


Then

$$
\lim _{t \rightarrow \infty} \mathrm{P}\{\|x(t)\|>\varepsilon\}=0
$$

for all $\varepsilon>0$.

Proof. Put $\zeta_{k}=\sup _{k \leq t \leq k+1}\|x(t)\|$. It is obvious that the result of the theorem follows if

$$
\lim _{k \rightarrow \infty} P\left\{\zeta_{k}>\varepsilon\right\}=0
$$

for all $\varepsilon>0$. By (3), (2), and Lemmas 1 and 2 we have

$$
\begin{aligned}
\mathrm{P}\left\{\zeta_{k}>\varepsilon\right\} \leq & \mathrm{P}\left\{\sup _{k \leq t \leq k+1}\left\|H_{0}^{t} x\right\|>(m+2)^{-1} \varepsilon\right\} \\
& +\mathrm{P}\left\{\sup _{k \leq t \leq k+1}\left\|\int_{0}^{t} H_{u}^{t} g_{0}(u) d u\right\|>(m+2)^{-1} \varepsilon\right\} \\
& +\sum_{j=1}^{m} \mathrm{P}\left\{\sup _{k \leq t \leq k+1}\left\|H_{0}^{t} \int_{0}^{t}\left(H_{0}^{u}\right)^{-1} g_{j}(u) d w_{j}(u)\right\|>(m+2)^{-1} \varepsilon\right\} \\
\leq & \left((m+2)^{-1} \varepsilon\right)^{-p} L_{0} D e^{-\lambda p k}\|x\|^{p} \\
& +\mathcal{L}_{3} \sum_{j=1}^{m} \sum_{i=0}^{k} e^{-(\lambda-\theta)(k-i) p\left(\sup _{i \leq u \leq i+1}\left\|g_{j}(u)\right\|\right)^{p}}
\end{aligned}
$$

for all $0<\theta<\lambda$.

Now we prove that

$$
\lim _{k \rightarrow \infty} \sum_{i=0}^{k} e^{-(\lambda-\theta)(k-i) p}\left(\sup _{i \leq u \leq i+1}\left\|g_{j}(u)\right\|\right)^{p}=0, \quad j=0, \ldots, m .
$$

Fix an arbitrary $\varepsilon>0$ and choose $n_{0} \in \mathbb{N}$ such that

$$
\max \left\{e^{-(\lambda-\theta) n p},\left(\sup _{n \leq u \leq n+1}\left\|g_{j}(u)\right\|\right)^{p}\right\}<\varepsilon \quad \text { for } n>n_{0} .
$$

Then

$$
\begin{aligned}
& \sum_{i=0}^{k} e^{-(\lambda-\theta)(k-i) p}\left(\sup _{i \leq u \leq i+1}\left\|g_{j}(u)\right\|\right)^{p} \\
& \quad=\sum_{i=0}^{n_{0}} e^{-(\lambda-\theta)(k-i) p}\left(\sup _{i \leq u \leq i+1}\left\|g_{j}(u)\right\|\right)^{p}+\sum_{i=n_{0}+1}^{k} e^{-(\lambda-\theta)(k-i) p}\left(\sup _{i \leq u \leq i+1}\left\|g_{j}(u)\right\|\right)^{p} \\
& \quad<e^{-(\lambda-\theta)\left(n_{0}+1\right) p}\left(\mathcal{M}_{j}\right)^{p} \sum_{i=0}^{\infty} e^{-(\lambda-\theta) p i}+\varepsilon \sum_{i=0}^{\infty} e^{-(\lambda-\theta) p i} \\
& \quad<\varepsilon\left(\left(\mathcal{M}_{j}\right)^{p}+1\right)\left(1-e^{-(\lambda-\theta) p}\right)^{-1}
\end{aligned}
$$

for all $k>2 n_{0}$, where $\mathcal{M}_{j}=\sup _{0 \leq u \leq+\infty}\left\|g_{j}(u)\right\|<+\infty$.

Theorem 1 is proved. 
Theorem 2. Let condition (2) hold and let $g_{i}(t), t \geq 0, i=0, \ldots, m$, be continuous functions such that

$$
\sum_{k=0}^{\infty} \sum_{i=0}^{k} e^{-\gamma(k-i) p}\left(\sup _{i \leq u \leq i+1}\left\|g_{j}(u)\right\|\right)^{p}<+\infty, \quad j=0, \ldots, m
$$

for some $p$ and $\gamma$ for which $0<p<p_{0}$ and $0<\gamma<\lambda$.

Then

$$
\lim _{t \rightarrow \infty} x(t)=0
$$

with probability one.

Proof. Let $\zeta_{k}=\sup _{k \leq t \leq k+1}\|x(t)\|$. The events $\lim _{t \rightarrow \infty} x(t)=0$ and $\lim _{k \rightarrow \infty} \zeta_{k}=0$ coincide. Therefore the result of the theorem follows if

$$
\sum_{k=0}^{\infty} \mathrm{P}\left\{\zeta_{k}>\varepsilon\right\}<+\infty
$$

for all $\varepsilon>0$ (see [1). An estimate of the general term of series (15) similar to that obtained in the proof of Theorem 1 implies Theorem 2 provided condition (14) is satisfied.

Theorem 2 is proved.

Let us consider the one-dimensional case in more detail. We find a rate of convergence to zero for power functions and this will imply the assumptions of Theorem 2 .

Consider the equations

$$
d y(t)=\left(b y(t)+(1+t)^{-l_{0}}\right) d t+\sum_{k=1}^{m}\left(\sigma_{k} y(t)+(1+t)^{-l_{k}}\right) d w_{k}(t) .
$$

We have

$$
\begin{gathered}
H_{s}^{t}=\exp \left\{-\gamma_{0}(t-s)+\sum_{k=1}^{m} \sigma_{k}\left[w_{k}(t)-w_{k}(s)\right]\right\}, \quad-\gamma_{0}=b-2^{-1} \sum_{k=1}^{m} \sigma_{k}^{2}, \\
\mathrm{E}\left|H_{s}^{t} x\right|^{p}=\exp \left\{\left(-\gamma_{0}+p 2^{-1} \sum_{k=1}^{m} \sigma_{k}^{2}\right)(t-s) p\right\}|x|^{p}, \\
p_{0}=\gamma_{0}\left(2^{-1} \sum_{k=1}^{m} \sigma_{k}^{2}\right)^{-1} .
\end{gathered}
$$

Condition (2) is satisfied for $\gamma_{0}>0$.

Theorem 3. Let $\gamma_{0}>0$ and let $l_{i}>p_{0}^{-1}, i=0, \ldots, m$. Then

$$
\lim _{t \rightarrow \infty} y(t)=0
$$

with probability one.

Proof. According to Theorem 2 it is sufficient to show that the series

$$
\sum_{k=0}^{\infty} \sum_{i=0}^{k} e^{-\gamma(k-i) p}(1+i)^{-l p}
$$


converges for some $p$ and $\gamma$ such that $0<p<p_{0}$ and $0<\gamma<\gamma_{0}$ if $l>p_{0}^{-1}$. The convergence of series (16) follows from the following upper bound for its general term:

$$
\begin{aligned}
\sum_{i=0}^{k} e^{-\gamma(k-i) p}(1+i)^{-l p}=(1+k)^{-l p} \sum_{i=0}^{k} e^{-\gamma(k-i) p} \frac{(1+k)^{l p}}{(1+i)^{l p}} & =(1+k)^{-l p} e^{2 \gamma p} \sum_{i=0}^{k} \exp p\left\{-\gamma(2+k-i)+l \ln \frac{(1+k)}{(1+i)}\right\} \\
\leq & (1+k)^{-l p} e^{2 \gamma p} \sum_{i=0}^{k} \exp p\{-\gamma(2+k-i)+l \ln (2+k-i)\} \\
\leq & (1+k)^{-l p} e^{2 \gamma p} \sum_{j=0}^{k} \exp p\{-\gamma(2+j)+l \ln (2+j)\} \leq \mathcal{A}(1+k)^{-l p},
\end{aligned}
$$

where

$$
\mathcal{A}=e^{2 \gamma p} \sum_{j=1}^{\infty} \exp p\{-\gamma j+l \ln j\}<+\infty .
$$

Theorem 3 is proved.

\section{BIBLIOGRAPHY}

1. I. I. Gikhman, A. V. Skorokhod, and M. I. Yadrenko, Probability Theory and Mathematical Statistics, "Vyshcha Shkola", Kyiv, 1979. (Russian)

2. N. Ikeda and S. Watanabe, Stochastic Differential Equations and Diffusion Processes, Second edition, North-Holland Publishing Company, Amsterdam, 1989. MR.1011252 (90m:60069)

3. A. V. Il'chenko, Stochastically bounded solutions of a linear inhomogeneous stochastic differential equation, Teor. Imovir. Mat. Stat. 68 (2003), 37-43; English transl. in Theory Probab. Math. Statist. 68 (2004), 48-55. MR2000393 (2004d:60150)

4. A. V. Il'chenko, Stochastically bounded solutions of the linear inhomogeneous stochastic differential equation system, Theory Stoch. Process. 9(25) (2003), no. 1-2, 65-72. MR2080014 (2005h:60166)

5. R. Z. Khasminskiı̌, Stochastic Stability of Differential Equations, "Nauka", Moscow, 1969; English transl., Sijthoff \& Noordhoff, Alphen aan den Rijn-Germantown, Md., 1980. MR600653 (82b:60064)

6. A. M. Sadovyak and E. F. Car'kov, An analogue of the Cauchy formula for stochastic differential equations, Teor. Veroyatnost. i Primenen. 18 (1973), no. 2, 415-417; English transl. in Theory Probab. Appl. 18 (1973), no. 2, 394-396. MR0319270 (47:7814)

7. A. V. Skorokhod, Asymptotic Methods in the Theory of Stochastic Differential Equations, "Naukova dumka", Kiev, 1987; English transl., American Mathematical Society, Providence, RI, 1989. MR:1020057 (90i:60038)

Department of General Mathematics, Faculty for Mechanics and Mathematics, National Taras Shevchenko University, Academician Glushkov Avenue 6, Kyiv 03127, Ukraine

Received 11/OCT/2005

Translated by S. KVASKO 